\title{
Analysis of daily activity data from implanted cardiac defibrillators: The minimum clinically important difference and relationship to mortality/life expectancy
}

\author{
Michael J. Shoemaker ${ }^{1 *}$, Amy B. Curtis ${ }^{1}$, Eric Vangsnes ${ }^{2}$, Michael G. Dickinson ${ }^{3}$, Rajib Paul ${ }^{4}$ \\ ${ }^{1}$ Interdisciplinary Health Sciences PhD Program, Western Michigan University, Kalamazoo, USA \\ ${ }^{2}$ Physician Assistant Program, Western Michigan University, Kalamazoo, USA \\ ${ }^{3}$ Spectrum Health Frederik Meijer Heart and Vascular Institute, Grand Rapids, USA \\ ${ }^{4}$ Department of Statistics, Western Michigan University, Kalamazoo, USA \\ Email: ${ }^{*}$ shoemami@gvsu.edu
}

Received 13 May 2012; revised 15 June 2012; accepted 27 June 2012

\section{ABSTRACT}

Background: Daily activity is a potentially important measure for assessing prognosis in individuals with chronic heart failure (CHF), and few studies have investigated the prognostic value of daily activity measurement. The present study sought to determine whether there is an association between daily activity and mortality/mean life expectancy as predicted by the Seattle Heart Failure Model (SHFM), and to provide an estimate of the anchor-based minimum clinically important difference (MCID) for daily activity measured by single-axis accelerometers in implanted cardiac defibrillators. Methods: This study utilized a retrospective chart review of 102 medical records of patients with CHF and Medtronic ${ }^{\circledR}$ implanted cardiac defibrillators (ICDs). Mean daily activity was calculated for a two week period prior to both a baseline and follow-up routine clinical visit. Clinical characteristics from the baseline clinic visit were used for calculating SHFM estimates of mean life expectancy, 1-year mortality, and 5-year mortality. A five-point global rating scale was scored based on documented clinician impression, patient self-report, and laboratory and cardiographic tests for determining the MCID. Results: There was a moderate correlation between baseline daily activity and each of the SHFM prognostic indicators: 1-year mortality $(r=0.36, p<$ 0.001 , 5-year mortality $(r=0.40, p<0.001)$, and life expectancy $(r=0.43, p<0.001)$. The MCID for a decline in daily activity was approximately 0.5 hours and was approximately 1.0 hours for improvement in daily activity. Conclusions: Although previous research has established the short-term predictive value of ICD-measured daily activity for CHF-related clinical events, no prior study has examined the longer-

${ }^{*}$ Corresponding author. term prognostic value of ICD-based daily activity. The results of the present study suggest that low daily activity, as recorded by ICDs in patients with CHF, should prompt a more formal evaluation of prognosis using the SHFM. Furthermore, changes of 0.5 to 1.0 hours of activity per day appear to be clinically meaningful.

Keywords: Heart Failure; Daily Activity; Prognosis; Cardiac Defibrillator

\section{INTRODUCTION}

Individuals with chronic heart failure $(\mathrm{CHF})$ are characterized by reduced daily physical activity, which is believed to contribute to a cycle of inactivity and deconditioning [1-9] and to reductions in exercise tolerance following the cessation of an exercise program [10,11]. Although daily activity has been used as an endpoint in several intervention-based studies [7,10-14], little data exists about the prognostic value of daily activity in heart failure. Walsh et al. [15] found that daily activity, measured using a pedometer dichotomized at $250 \times 10^{2}$ steps per week, was an independent predictor of mortality in a multivariate analysis of 84 subjects with CHF. More recently (2010), in a sample of 60 subjects with CHF, Howell et al. [16] reported that subjects with a peak daily activity level of less than 22 metabolic equivalents per minute (measured using actigraphy) had lower 11-month, event-free survival. The devices used to measure daily activity in the aforementioned studies, however, are external devices that depend on subjects' adherence, and neither study provided data regarding the amount of change in daily activity that is considered to be clinically meaningful. The single-axis accelerometers in implanted cardiac defibrillators (ICDs) avoid the potential problems 
of subject non-adherence and selection bias in studies examining daily activity in patients with CHF. No prior study has investigated whether this implanted devicebased measure of daily activity is associated with life expectancy and mortality, nor has any prior study investigated what amount of change in this measure is considered to be clinically meaningful. These are the primary aims of the present study.

Medtronic ${ }^{\circledR}$ ICDs (Medtronic, Minneapolis, MN) contain a single-axis accelerometer that, on a minute-tominute basis, determines whether patient activity exceeded an equivalent of 70 - 80 steps/minute. The device then records the number of minutes of activity on a daily basis, which is stored for a rolling 14 month period [17]. Among the various methods for measuring daily activity, including questionnaires, measurement of energy expenditure, and external accelerometers [18], ICD-based daily activity measures are not limited by short monitoring periods, monitor wearing non-adherence, and patient recollection. Despite these advantages, few studies have utilized this measure [19-21].

The daily activity data recorded by Medtronic ${ }^{\circledR}$ implanted ICDs (labeled "patient activity" by Medtronic ${ }^{\circledR}$ ) have been examined in three studies. Gulati et al. [19] found that the six-minute walk test (6MWT) and daily activity measured within the preceding or subsequent 24 hours of conducting the test were moderately correlated $(r=0.42$ and 0.49 , respectively). Despite their findings of only moderate correlation, the authors concluded that daily activity may be considered a surrogate for the 6 MWT. Both Adamson et al. [20] and Small et al. [21] considered daily activity among a variety of devicebased parameters for predicting hospitalization for acute decompensated heart failure. Only Adamson et al. [20] found that a decline in daily activity was a statistically significant predictor, with a decline of 0.4 hours per day in activity between baseline and time of hospitalization. Daily activity may not have been a significant predictor in the analysis by Small et al. [21] because they dichotomized daily activity at 0.5 hours per day. No prior study has investigated the amount of change in ICDmeasured daily activity that is considered to be clinically meaningful.

The minimum clinically important difference (MCID) is the smallest change that a patient would perceive as beneficial, and when considering a decline in the measure of interest, would indicate the need for a change in treatment $[22,23]$. Calculation of the MCID includes distribution-based and anchor-based methods [23]. Distribution-based methods include calculation of an effect size which provides a ratio of change in an outcome to the baseline standard deviation of that measure for interpreting whether the effect observed in a given trial was statistically significant, while anchor-based methods de- termine the mean amount of change associated with perceived level of change using the Global Rating of Change Scale (GRS). The anchor-based MCID can utilize either a patient-based or clinician-based anchor [25]. A clinician-based anchor in addition to other objective clinical data may be more appropriate for judging change in disease status [24].

The only two studies to report on the relationships between daily activity and prognosis in individuals with CHF were longitudinal studies that measured mortality directly $[15,16]$. Howell et al. [16] followed subjects for up to one year and Walsh et al. [15] up to five years. Although direct measurement of mortality is ideal, it is difficult to study design to implement. The Seattle Heart Failure Model (SHFM) is a multivariate risk model developed based on data from six published pharmacologic intervention clinical trials representing nearly 11,000 patients with systolic heart failure [24]. The model predicts four primary outcomes: 1,3 , and 5-year mortality and mean life expectancy. The 16 independent predictors in the model include data related to patient characteristics, medications, laboratory results, and use of implanted interventional devices. No prior study has examined whether daily activity is associated with the SHFM prognostic indicators.

In summary, daily activity is a potentially important measure for assessing prognosis in individuals with CHF. Further, it is not known whether daily activity is associated with the SHFM prognostic estimates and what amount of change in daily activity is necessary to be considered clinically meaningful. Therefore, the purpose of the present study was two-fold: 1) To determine whether there is an association between daily activity and mortality/mean life expectancy as predicted by the Seattle Heart Failure Model, and 2) to provide an estimate of the anchor-based MCID for daily activity measured by single-axis accelerometers in implanted devices.

\section{METHODS}

\subsection{Design}

Retrospective chart review of clinical data at two time points at least three months apart between January 2008 and January 2011 in patients with Medtronic ${ }^{\circledR}$ ICDs.

\subsection{Subjects}

One hundred thirty-five medical records (approximately two-thirds of available records) were randomly selected from a list of patients with Medtronic ${ }^{\circledR}$ ICDs who were managed by a tertiary heart failure and transplant services clinic between January 2008 and January 2011. Medical records were included if the patient had a Medtronic implanted device (ICD or biventricular ICD) 
and a diagnosis of systolic heart failure with a history of LVEF $<40 \%$. Records were excluded if there was insufficient data in the medical record to achieve the study objectives described below.

\subsection{Clinical Data Extraction}

Clinical data representing two points in time for each patient were extracted. Time point 1 was a clinic visit for which daily activity data was available for the preceding two weeks and that provided the necessary clinical and laboratory data for the SHFM. Time point 2 was a subsequent clinic visit at least three months after time point 1 for which daily activity data was available in the preceding two weeks and had sufficient documentation for determining whether or not there was a change in clinical status. Extracted data included the variables required for the SHFM, patient and clinician quotes regarding change in clinical status between the two time points, and device-recorded daily activity.

\subsection{Measurements}

The anchor used to calculate the MCID was the 5-point GRS. The GRS captured change in clinical status using the clinician's documented impression of the course of the patient's overall disease state as supported by documented patient self-report and changes in the patient's clinical characteristics. These clinical characteristics included left ventricular ejection fraction, New York Heart Association (NYHA) Functional Class, B-type natriuretic peptide, hospitalization or other significant health event, and change in CHF-related medications.

Medtronic $^{\circledR}$ ICDs contain a single-axis accelerometer that record the amount of daily activity every 24 hours for a rolling 14-month period. The number of hours of activity in each 24 hour period is based on the number of minutes per day in which the patient was active at or above an equivalent of 70 - 80 steps/min, with each minute recorded as a dichotomized value of yes/no [17]. Daily activity is plotted for a 14-month period on the printed report generated by the Cardiac Compass ${ }^{\circledR}$ device summary. The data point included for analysis was a visual estimate of the mean activity (hours per day) for the 2 weeks preceding the clinic visit on which the time point is based. Daily activity data were not measured/ extracted until after other data, including the GRS, were collected. To ensure accuracy of visually estimating the mean daily activity from the activity graph, two researchers independently estimated the mean daily activity for 25 separate sample cases with a range of 0 to 8 hours of activity per day. The intra-class correlation coefficient $(2,1)$ was 0.998 (95\% confidence interval 0.996 to 0.999$)$.

The SHFM prognostic estimates for life expectancy, 1-year mortality, and 5-year mortality were calculated using the extracted clinical data from time point 1 . Three laboratory values not routinely collected on each clinical visit that are required for the SHFM were total cholesterol, lymphocyte percentage, and uric acid. Therefore, if the lab value was collected within six months of the clinic visit, this value was recorded and attributed to the baseline dataset. Using this strategy, total cholesterol and uric acid were missing in 4 and 42 cases, respectively. These values were imputed using the aregImpute multiple imputation function from Harrell's Hmisc package [26] as used by Gorodeski et al. [27] who were missing similar data for use in the SHFM.

\subsection{Data Analysis}

\subsubsection{Association between Daily Activity and Mortality/Life Expectancy}

The Pearson correlation coefficient was calculated for daily activity at time point 1 (hours per day) and each of the following predicted values derived from the SHFM: 1 year mortality (\%), 5 year mortality $(\%)$, and mean life expectancy (years).

\subsubsection{Determination of the Anchor-Based MCID}

Change in daily activity between time points 1 and 2 was used to determine the anchor-based MCID for this measure. One-way analysis of variance with repeated planned contrasts was used to compare the mean change in daily activity for each category of the GRS to the previous one. The mean and 95\% confidence interval for change in daily activity between time points 1 and 2 for each level of the GRS were used to identify whether there was an apparent threshold of change in daily activity for those with and without change in clinical status. The magnitude of the MCID was contextualized using the NYHA Functional Classification. Classes II and III/ IV represent distinct symptomatic clinical presentations and have previously been shown to have statistically significantly different aerobic capacities [28], and the difference in daily activity between these classes may provide insight into what amount of change in daily activity is clinically meaningful. Therefore, daily activity for Classes II and III/IV were compared using t-tests.

\section{RESULTS}

Of the 135 medical records reviewed, 33 records were excluded due to not having at least two clinic visits and/ or not having a Cardiac Compass ${ }^{\circledR}$ report available for extracting daily activity data. Descriptive statistics for the overall sample of 102 included cases are presented in Table 1. There was a moderate correlation between baseline daily activity and each of the SHFM prognostic indicators: 1-year mortality $(r=0.36, p<0.001,5$-year 
Table 1. Descriptive statistics.

\begin{tabular}{cc}
\hline & Mean (SD) \\
\hline Age & $64.6(13.3)$ years \\
Gender & 30 females, 72 males \\
Etiology & 50 ischemic, 52 nonischemic \\
NYHA-FC & $2.0(0.76)$ \\
LVEF & $27.8(11.5) \%$ \\
Type of Device & $26 \mathrm{ICD}, 72 \mathrm{BiVICD}$ \\
Time between Visits & $7.5(4.2)$ months \\
Daily Activity & $2.6(1.7)$ hours $\cdot$ day \\
-1 \\
Life Expectancy & $10.1(5.2)$ years \\
1 Year Mortality & $9.4(9.3) \%$ \\
5 Year Mortality & $33.0(24.1) \%$ \\
\hline
\end{tabular}

NYHA-FC $=$ New York Heart Association Functional Class; LVEF $=$ left ventricular ejection fraction; $\mathrm{ICD}=$ implantable cardiac defibrillator; $\mathrm{BiVICD}=$ biventricular implantable cardiac defibrillator.

mortality $(\mathrm{r}=0.40, \mathrm{p}<0.001)$, and life expectancy $(\mathrm{r}=$ $0.43, \mathrm{p}<0.001)$. Figure 1 depicts the scatterplot for daily activity and life expectancy.

With regard to the MCID for ICD-measured daily activity, descriptive statistics for cases stratified by GRS are presented in Table 2 . There was a statistically significant main effect of GRS on change in daily activity $[\mathrm{F}(4,97)=21.48, \mathrm{p}=0.000, \omega=0.41]$. Planned contrasts revealed statistically significant differences $(\mathrm{p}<0.05)$ between each subsequent GRS category except between "about the same" and "a little better" $(\mathrm{p}=0.085)$. Figure 2 demonstrates the mean and $95 \%$ confidence interval for change in daily activity for each GRS category. The MCID for a decline in daily activity is approximately 0.5 hours and is approximately 1.0 hours for improvement in daily activity. There was a statistically significant mean difference of 0.88 hours of baseline activity between NYHA classes II and II/IV [2.7 (1.8) hours for those with NYHA class II versus $1.8(1.5)$ hours for those with class $\mathrm{III} / \mathrm{IV}, \mathrm{t}(1,73)=2.24, \mathrm{p}=0.014$, effect size $=0.54]$.

\section{DISCUSSION}

Daily activity is a potentially important measure of prognosis in individuals with CHF, and no prior published study has reported what amount of change in daily activity is considered to be clinically meaningful. The results of the present study demonstrated that ICD-measured daily activity was associated with disease severity as measured by the SHFM, and changes of 0.5 and 1.0 hours of daily activity appear to represent clinically meaningful decline and improvement, respectively.

With regard to the moderate correlation between daily activity and the SHFM, daily activity was more strongly associated with life expectancy than 1- and 5-year mortality, explaining $19 \%$ of the variance in calculated life

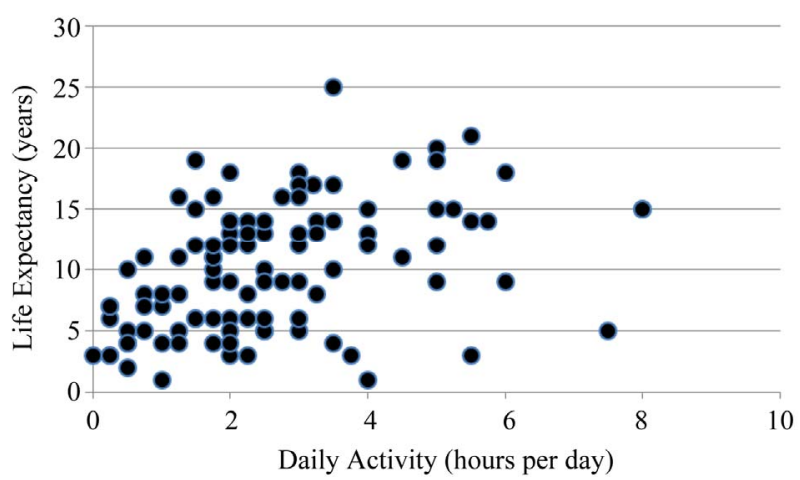

Figure 1. Scatterplot of baseline daily activity versus life expectancy. $\mathrm{r}=0.43, \mathrm{p}<0.001$.

expectancy. Implanted device-measured daily activity has previously been demonstrated to be a useful predictor of hospitalization for acute decompensated heart failure [20], and daily activity measured using actigraphy and pedometers was predictive of mortality $[15,16]$. The results of the present study suggest that, in addition to consideration of other prognostic factors including the SHFM, ICD-based measurement of daily activity may also be clinically useful in identifying individuals with increased risk of mortality and decreased life expectancy. This research did not address whether an intervention to improve daily activity would result in a change in prognosis, and this should be addressed by future research.

With regard to the MCID for daily activity, a lesser amount of change in daily activity was associated with a clinically meaningful decline compared to the amount of change representing clinically meaningful improvement. There are two potential explanations for this difference. First, although not previously reported for daily activity, two prior studies $[29,30]$ found that the MCID for improvement and decline in the 6MWT in individuals with CHF were dissimilar. Second, and perhaps more likely, there was no statistically significant difference in daily activity between those cases rated as "about the same" and "a little better" because this analysis had less statistical power (effect size $=0.43,1-\beta=0.38$ ) than did the analysis of those cases rated as "about the same" and "a little worse" (effect size $=0.55,1-\beta=0.55$ ) due to a smaller mean difference.

There are two considerations for contextualizing the magnitude of these MCID estimates. First, Small et al. [21] found a mean decline of 0.4 hours in those patients hospitalized for acute decompensated heart failure, which likely represents a clinically meaningful change and is consistent with the results of the present study. Additionally, the mean difference of 0.88 hours in the present study between those with NYHA class II and class III/IV, although not suggestive of a direction of change (e.g., improvement vs. decline), also suggests that a meaningful difference in daily activity is $\leq 1.0$ 
Table 2. Descriptive statistics stratified by global rating scale category.

\begin{tabular}{|c|c|c|c|c|c|}
\hline & $\begin{array}{c}\text { Much Worse } \\
n=10 \\
\text { Mean (SD) }\end{array}$ & $\begin{array}{c}\text { A Little Worse } \\
n=13 \\
\text { Mean (SD) }\end{array}$ & $\begin{array}{c}\text { About the Same } \\
\mathbf{n}=57 \\
\text { Mean (SD) }\end{array}$ & $\begin{array}{c}\text { A Little Better } \\
n=12 \\
\text { Mean (SD) }\end{array}$ & $\begin{array}{c}\text { Much Better } \\
\mathrm{n}=10 \\
\text { Mean (SD) }\end{array}$ \\
\hline Age [years] & $69.8(7.4)$ & $62.3(17.0)$ & $64.8(12.4)$ & $64.4(18.4)$ & $62.0(11.4)$ \\
\hline Gender [\% male] & 90.0 & 61.5 & 71.9 & 66.7 & 60.0 \\
\hline Etiology [\% ischemic] & 50.0 & 69.2 & 49.1 & 25.0 & 50.0 \\
\hline LVEF [\%] & $26.5(10.6)$ & $24.5(5.3)$ & $31.0(12.4)^{*}$ & $26.3(9.3)$ & $17.5(8.9)^{*}$ \\
\hline NYHA-FC & $2.0(0.8)$ & $1.8(0.6)$ & $1.9(0.8)$ & $2.2(0.7)$ & $2.9(0.7)$ \\
\hline 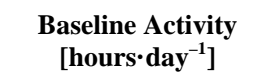 & $2.6(2.2)$ & $2.9(1.5)$ & $2.7(1.6)$ & $2.7(2.4)$ & $1.9(1.8)$ \\
\hline $\begin{array}{l}\text { Change in Activity } \\
\text { [hours· day }{ }^{-1} \text { ] }\end{array}$ & $-1.3(0.7)$ & $-0.52(0.7)^{*}$ & $0.04(0.6)^{*}$ & $0.4(0.6)$ & $1.08(0.7)^{*}$ \\
\hline
\end{tabular}

*one-way ANOVA repeated contrast $\mathrm{p}<0.05$ compared to previous level; LVEF $=$ left ventricular ejection fraction, NYHA-FC $=$ New York Heart Association Functional Class.

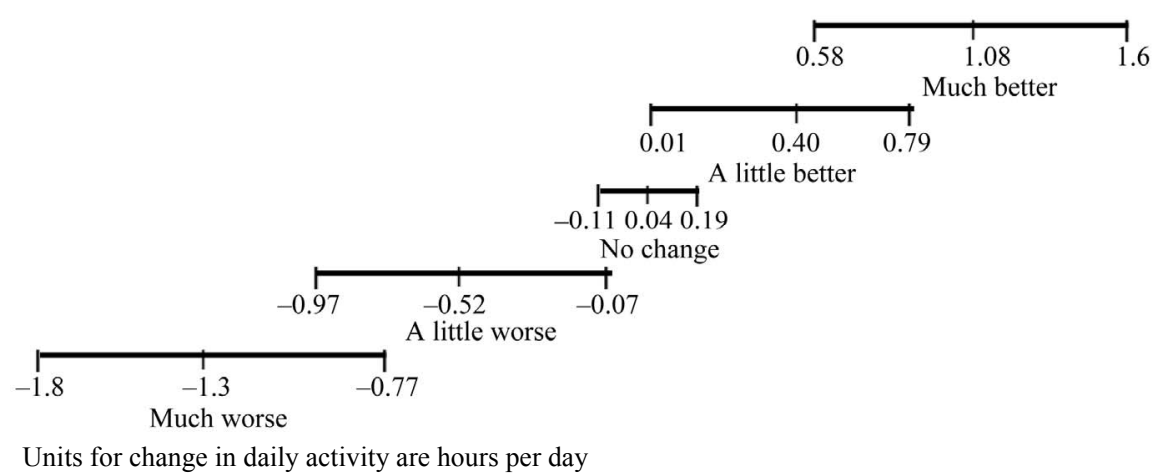

Figure 2. Mean and 95\% confidence interval for change in daily activity stratified by global rating scale.

hours. Other indices of clinically meaningful change for ICD-measured daily activity should be examined in future research. For example, the minimum detectable difference (an index of test-retest reliability or measurement error), would provide an estimate of the amount of variation in daily activity that represents background "noise," against which changes in daily activity could be compared.

\subsection{Limitations}

There are several limitations of the present analyses. First, the anchor used for the MCID analysis was based on clinician impression as abstracted from clinical documentation, which was not intended for such an analysis. However, clinician-based anchors have been reported by some to represent a better anchor than patient perception-based anchors [25]. The present study used the clinician's impression of change in clinical status as supported by a wide range of supporting clinical data. Second, uric acid had to be imputed in 42 cases, nearly one third of the overall data set. However, the method used for imputation has been previously demonstrated for in a greater percent of cases for this variable for use in the
SHFM [27]. Third, the findings of the present paper may not be generalizable to other types and brands of cardiac defibrillators as the measurement, processing, and reporting of activity data by other devices may be substantially different than Medtronic ${ }^{\circledR}$ devices. Fourth, not all patient characteristics were similar when stratified according to the GRS. For example, there was a greater proportion of males in the "much worse" group and a lower mean LVEF in the "much better" group. The influence of the differences on the results is not clear. Finally, daily activity was measured using a visual estimate from a printed graph using a two-week sample preceding each clinical visit. Although this method demonstrated excellent inter-rater reliability, a more accurate method for quantifying daily activity is needed. Additionally, use of longer sampling frame that allows for characterization of the trend of daily activity (i.e., improving, declining, fluctuating, or stable) prior to calculation of the SHFM prognostic indicators may reveal a greater prognostic value for daily activity.

\subsection{Conclusion}

Daily activity as recorded by ICDs in patients with CHF 
appears to be a promising new measure for the research and clinical settings. Low daily activity, as recorded by ICDs in patients with CHF, should prompt a more formal evaluation of prognosis using the SHFM. Furthermore, changes of 0.5 to 1.0 hours of activity per day appear to be clinically meaningful.

\section{ACKNOWLEDGEMENTS}

The authors thank the clinical staff at the Spectrum Health Cardiac Device Clinic.

\section{REFERENCES}

[1] Oka, R.K., Gortner, S.R., Stotts, N.A. and Haskell, W.L. (1996) Predictors of physical activity with chronic heart failure secondary to either ischemic or idiopathic dilated cardiomyopathy. American Journal of Cardiology, 77, 159-163. doi:10.1016/S0002-9149(96)90588-3

[2] Van den Berg-Emons, H., Bussmann, J., Balk, A., et al. (2001) Level of activities associated with mobility during everyday life in patients with chronic congestive heart failure as measured with an "Activity Monitor." Physical Therapy, 81, 1502-1511.

[3] Davies, S.W., Jordan, S.L. and Lipkin, D.P. (1992) Use of limb movement sensors as indicators of the level of everyday physical activity in chronic congestive heart failure. American Journal of Cardiology, 69, 1581-1586. doi:10.1016/0002-9149(92)90707-6

[4] Hoodless, D.J., Stainer, K., Savic, N., et al. (1994) Reduced customary activity in chronic heart failure: Assessment with a new shoe-mounted pedometer. International Journal of Cardiology, 43, 39-42. doi:10.1016/0167-5273(94)90088-4

[5] Walsh, J.T., Andrews, R., Evans, A. and Cowley, A.J. (1995) Failure of "effective" treatment for heart failure to improve customary activity. British Heart Journal, 70, 373-376. doi:10.1136/hrt.74.4.373

[6] Toth, M.J., Gottlieb, S.S., Goran, M.I., et al. (1997) Daily energy expenditure in free-living heart failure patients. American Journal of Physiology, 272, E469-E475.

[7] van den Berg-Emons, R., Balk, A., Bussmann, H. and Stam, H. (2004) Does aerobic training lead to a more active lifestyle and improved quality of life in patients with chronic heart failure? European Journal of Heart Failure, 6, 95-100. doi:10.1016/i.ejheart.2003.10.005

[8] Oka, R.K., Stotts, N.A., Dae, M.W., et al. (1993) Daily physical activity levels in congestive heart failure. American Journal of Cardiology, 71, 921-925. doi:10.1016/0002-9149(93)90907-T

[9] Witham, M.D., Argo, I.S., Johnston, D.W., Struthers, A.D. and McMurdo, M.E.T. (2006) Predictors of exercise capacity and everyday activity in older heart failure patients. European Journal of Heart Failure, 8, 203-207. doi:10.1016/j.ejheart.2005.03.008

[10] Willenheimer, Rydberg, E., Cline, C., et al. (2001) Effects on quality of life symptoms, and daily activity 6 months after termination of an exercise programme in heart fail- ure patients. International Journal of Cardiology, 77, 2531. doi:10.1016/S0167-5273(00)00383-1

[11] Witham, M.D., Gray, J.M. and Argo, I.S. (2005) Effect of a seated exercise program to improve physical function and health status in frail patients $>70$ years of age with heart failure. American Journal of Cardiology, 95, 11201124. doi:10.1016/j.amjcard.2005.01.031

[12] Mueller, L., Myers, J., Kottman, W., et al. (2007) Exercise capacity, physical activity patterns and outcomes six years after cardiac rehabilitation in patients with heart failure. Clinical Rehabilitation, 21, 923-931. doi:10.1177/0269215507079097

[13] Gottlieb, S.S., Fisher, M.L., Freudenberger, R., et al. (1999) Effects of exercise training on peak performance and quality of life in congestive heart failure patients. Journal of Cardiac Failure, 5, 188-194. doi:10.1016/S1071-9164(99)90002-7

[14] Walsh, J.T., Andrews, R., Evans, A. and Cowley, A.J. (1995) Failure of "effective" treatment for heart failure to improve normal customary activity. British Heart Journal, 73, 373-376. doi:10.1136/hrt.74.4.373

[15] Walsh, J.T., Charlesworth, A., Andrews, R., Hawkins, M. and Cowley, A.J. (1997) Relation of daily activity levels in patients with chronic heart failure to long-term prognosis. American Journal of Cardiology, 79, 1364-1369. doi:10.1016/S0002-9149(97)00141-0

[16] Howell, J., Strong, B.M., Weisenberg, J., et al. (2010) Maximum daily 6 minutes of activity: An index of functional capacity derived from actigraphy and its application to older adults with heart failure. Journal of the American Geriatric Society, 58, 931-936. doi:10.1111/j.1532-5415.2010.02805.x

[17] Allison, G.-C. (2009) Principal Scientist, Medtronic Corporation.

[18] Casburi, R. (2009) Activity monitoring in assessing activities of daily living. COPD, 4, 251-255. doi:10.1080/15412550701480158

[19] Gulati, S.K., McKenzie, J., Crossley, G., Papp, M.A., Sims, J. and Andrulli, J. (2009) Device measured physical activity: Is it the new 6-minute hall walk? Journal of Cardiac Failure, 15, S119-120. doi:10.1016/j.cardfail.2009.06.055

[20] Adamson, P.B., Smith, A.L., Abraham, W.T., et al. (2004) Continuous autonomic assessment in patients with symptomatic heart failure: Prognostic value of heart rate variability measured by an implanted cardiac resynchronization device. Circulation, 110, 2389-2394. doi:10.1161/01.CIR.0000139841.42454.78

[21] Small, R.S., Wickemeyer, W., Germany, R., et al. (2009) Changes in intrathoracic impedance are associated with subsequent risk of hospitalizations for acute decompensated heart failure: Clinical utility of implanted device monitoring without a patient alert. Journal of Cardiac Failure, 15, 475-481. doi:10.1016/j.cardfail.2009.01.012

[22] Jaeschke, R., Singer, J. and Guyatt, G.H. (1989) Measure of health status: Ascertaining the minimal clinically important difference. Controlled Clinical Trials, 10, 407415. doi:10.1016/0197-2456(89)90005-6

[23] Lydlick, E. and Epstein, R.S. (1993) Interpretation of 
quality of life measures. Quality of Life Research, 2, 221226. doi:10.1007/BF00435226

[24] Levy, W.C., Mozaffarian, D., Linker, D.T., et al. (2006) The Seattle Heart Failure Model: Prediction of survival in heart failure. Circulation, 113, 1424-1433. doi:10.1161/CIRCULATIONAHA.105.584102

[25] Beaton, D.E., Boers, M. and Wells, G.A. (2002) Many faces of the minimal clinically important difference (MCID): A literature review and directions for future research. Current Opinion in Rheumatology, 14, 109-114. doi:10.1097/00002281-200203000-00006

[26] HMISC: Harrell miscellaneous. http://cran.Rproject.Org/web/packages/hmisc/index.html

[27] Gorodeski, E.A., Chu, E.C., Chow, C.H., et al. (2003) Application of the Seattle Heart Failure Model in ambulatory patients presented to an advanced heart failure therapeutics committee. Circulation and Heart Failure, $\mathbf{3}$, 706-714.

doi:10.1161/CIRCHEARTFAILURE.110.944280

[28] Russell, D.S., Saval, M.A., Robbins, J.L., et al. (2009) New York Heart Association functional class predicts exercise parameters in the current era. American Heart Journal, 158, S24-S30. doi:10.1016/j.ahj.2009.07.017

[29] O'Keeffe, S.T., Lye, M., Donnellan, C. and Carmichael, D.N. (1998) Reproducibility and responsiveness of quality of life assessment and six minute walk test in elderly heart failure patients. Heart, 80, 377-382.

[30] Spertus, J., Peterson, E., Conard, M.W., et al. (2005) Monitoring clinical changes in patients with heart failure: A comparison of methods. American Heart Journal, 150, 707-715. doi:10.1016/j.ahj.2004.12.010 